(FC) is useful in differentiating inflammatory bowel disease (IBD) from non-organic functional bowel disorders. FC may also be useful in monitoring patients with IBD to identify acute flares in disease, long before the patient becomes symptomatic. This allows appropriate treatment to be given in situations where disease activity is objectively measured to be greater than any perceived deterioration in symptoms.

Methods Faecal calprotectin data was collected over a 2 year period from 110 patients with IBD, who underwent regular outpatient assessment. Results were recorded as normal $(<50 \mu \mathrm{g} / \mathrm{g})$, borderline $(50-100 \mu \mathrm{g} / \mathrm{g})$ and elevated $(>100 \mu \mathrm{g} / \mathrm{g})$. A retrospective analysis of management outcome was made from reviewing patient records on the local clinical correspondence store and the new National IBD-Registry.

Results 44, 5 and 61 patients had normal, borderline and elevated FC levels, respectively. Three patients with normal FC (6.8\%), compared to $29(47.5 \%)$ with elevated FC, required upregulation of their management for symptom control. FC returned to normal levels in those selected for treatment escalation. In total, 104/110 (94.5\%) of patients avoided investigative colonoscopy. Six patients did require colonoscopic assessment, 1 had normal FC, 1 was borderline and 4 had elevated FC. Three of these 6 colonoscopic examinations were performed as part of surveillance and 2 were carried out in FC +ive patient, 1 for planned therapeutic dilatation of a known stricture and the other to confirm the severe and extent of their disease prior to referral for surgical intervention.

Conclusion FC is a useful monitoring tool in IBD and in identifying those who have developed or are about to suffer an acute flare in their disease. This allows adjustment of treatment without having to subject patients to colonoscopic investigation. A positive result directs the need to upregulate management, whilst a negative one can be reassuring, allowing physician to concentrate on treating functional aspects if the patient is symptomatic. Disclosure of Interest None Declared.

\section{PTU-098 HIGH LEVELS OF EMOTIONAL AND PHYSICAL DISTRESS AMONG FAMILY CAREGIVERS OF INDIVIDUALS WITH INFLAMMATORY BOWEL DISEASE (IBD)}

K Argyriou*, A Kapsoritakis, S Potamianos. Gastroenterology, University Hospital of Thessaly, Larissa, Greece

\subsection{6/gutjnl-2014-307263.172}

Introduction In chronic debilitating disorders, caregiving can be stressful and may contribute to serious illness and depression. However, in less debilitating disorders, such as IBD, limited data are available about the impact of the disease on caregiver's wellbeing. Our aim was to identify the emotional and physical distress among family caregivers of IBD patients in order to provide preventive services to an at-risk but hidden population.

Methods Over a 19 month period, in the tertiary referral centre for IBD in Central Greece, we interviewed 44 family caregivers of Crohn's Disease (CD) and 41 of Ulcerative Colitis (UC) patients. Caregiver's distress was assessed by using the caregiver self-assessment questionnaire "How Are You ?" of the American Medical Association. Disease activity was assessed using the Harvey-Bradshaw index for $\mathrm{CD}$ and Simple Colitis Activity Index for UC. A card with the sociodemographic and clinical characteristics of the population were also collected. The impact of each characteristic on caregiver's well-being was studied with one and two way ANOVA.
Results $84,7 \%$ of the caregivers showed high levels of emotional and physical distress with the majority of them to be middleaged women (61.2\%) with basic educational level (61,2\%)and full-time employment $(65,9 \%)$ at the time of the study. No significant difference found on the level of distress between caregivers of CD and those of UC patients. Factor analysis identified that female gender, active disease, disease related complications and long lasting disease had significant impact on the caregiver's level of distress.

\section{Conclusion}

1. Family caregivers of IBD patients experience high levels of emotional and physical distress.

2. Active disease, disease related complications, long lasting disease and female gender have significant impact on caregiver's level of distress.

3. It is necessary to organise a network to provide psychosocial services for the caregivers of IBD patients.

\section{REFERENCES}

1 Epstein-Lubow G, et al. Evidence for the validity of the American Medical Association's caregiver self-assessment questionnaire as a screening measure for depression. J Am Geriatr Soc 2010 Feb;58(2):387-8

2 Epstein-Lubow Family caregiving during healthy aging and illness. Med Health $R$ I. 2012 Jul:95(7):222-3

Disclosure of Interest None Declared.

\section{PTU-099 SURVEILLANCE COLONOSCOPY IN INFLAMMATORY BOWEL DISEASE IN A DISTRICT GENERAL HOSPITAL - ARE WE IMPROVING?}

'L-L Clark*, ${ }^{2} S$ Paterson. 'Gastroenterology, Victoria Infirmary Glasgow, Glasgow, UK; ${ }^{2}$ Gastroenterology, Forth Valley Royal Hospital, Larbert, UK

\subsection{6/gutjnl-2014-307263.173}

Introduction Patients with inflammatory bowel disease (IBD) have an increased risk of colorectal cancer. In 2009 the British Society of Gastroenterology updated guidelines recommending chromoendoscopy with targeted biopsies of suspicious areas, Grade A; or 2-4 biopsies every $10 \mathrm{~cm}$, Grade C. A local audit in Forth Valley Royal Hospital (FVRH) looking at adherence to guidelines was carried out in 2009 showing adherence to guidelines in $27 \%(12 / 44)$ of cases. Subsequent to this, information regarding the current guidelines was disseminated to clinicians and three medical consultants took responsibility for performing chromoendoscopy. Practice was then re-audited.

Methods FVRH is a district general hospital with a catchment area of 300,000. The endosocpy reporting tool Unisoft was used to identify patients undergoing colonoscopy with the indication "follow up - colitis surveillance" during a 1 year period from 01/01/2012. The endoscopy report was reviewed noting the endoscopist, completeness of procedure, and adherence to a surveillance guideline. The pathology reports were accessed via the SCI store computer system.

Results 87 patients were identified (44 identified over 6 months during the previous audit) as having colitis surveillance colonoscopy. $60(69 \%)$ procedures adhered to one of the recognised surveillance strategies (37 dye-spray, 23 biopsy protocol) compared to $27 \%(12 / 44)$ in 2009 . There has also been a marked increase in the number of patients who had chromoendoscopy; 43\% (37/87) versus $4.5 \%(2 / 44)$ in 2009 . The other main difference in this audit was the type of endoscopist performing the procedure (medical $70 \%$ v $32 \%$, nurse $22 \%$ v $43 \%$, surgeon $25 \%$ v $8 \%$ ). Medical gastroenterologists performed 34 of the 37 chromoendoscopic 